\title{
Predictors of Outcome in Drug Resistant Tuberculosis Patients
}

\section{Arivudainambi Periasamy*}

K.A.P.Viswanatham Government Medical College, Tiruchirappalli, India

\begin{abstract}
Introduction: Multi-drug-resistant tuberculosis (MDRTB) is a major public health problem due to longer duration of treatment and unfavourable outcome in comparison with sensitive TB.
\end{abstract}

Aim: Registered Drug resistant tuberculosis (DRTB) patients were prospectively followed from 2011-2015. Analysis of treatment outcomes was by done by their demographic and clinical data which were hypothesized to be outcome predictors.

Methods: Logistic regression and univariate logistic regression were used upon data of patients registered for MDRTB treatment in Trichy district Tamilnadu, India. Totally 63 MDRTB patients were treated from 2011-15. Outcomes were reported as success \& failure. Success included cured and treatment completed patients and Failure included treatment failure, death, defaulted, untraceable cases. SPSS 21 was used.

Results: Out of total 63 cases, 33 cases had failed outcome and 30 cases had successful outcome. Among variables Diabetes, XDRTB suspects, drug abuse \& smoking were associated with failed outcome. Sex, age, chest $x$-ray lesion, pre-treatment resistance profile, did not affect the outcome. By logistic regression (enter) method the odds of failed outcome were 11.737 with smoking, the odds of failed outcome was 12.43 with XDRTB suspects, and the odds of failure with diabetes was 12.61. The P-value of smoking, XDRTB suspect, diabetes obtained was 0.019 , 0.049 , and 0.036 respectively. Chi square test showed significant $P$-value for variables sex, diabetes, XDR suspects, smoking and drug abuse, but insignificant P-value for HIV, comorbid illness like renal failure. Similarly, age, chest $\mathrm{x}$-ray lesion like cavitary, caseous lesion, did not affect the outcome as per logistic regression analysis.

Conclusion: Smoking, PreXDRTB, Diabetes, were factors affecting the outcome of treatment independently hence predictors of outcome in MDRTB. The emergence of preXDRTB as independent factor determining the outcome is significant emphasizing the earlier switch to XDRTB regimen.

Keywords: MDRTB; TB and diabetes; XDRTB; TB mortality

Abbreviations: DRTB: drug resistant tuberculosis; MDRTB: Multi drug resistant tuberculosis; DM: Diabetes mellitus; CXR: Chest X-Ray.

\section{Background}

Drug resistant tuberculosis (DRTB) included both multidrugresistant (MDR) and extensively drug-resistant (XDR) TB; MDRTB strains are resistant to Isoniazid and Rifampicin the two most-effective first-line any of the injectables [1]. Without improvement in diagnosis and treatment for DRTB, MDRTB and XDRTB could become the dominant forms of TB worldwide [2]. Drug resistant tuberculosis (DRTB) is a global health problem affecting the progress from control to elimination [2]. Resistant to Isoniazid, rifampicin that forms the back bone of the short course chemotherapy would require use of drugs that are more toxic, costly and that administered for long period [3-5]. Among the Proportion of TB cases with drug resistance: about $3.7 \%$ of new tuberculosis (TB) patients in the world have multidrugresistant strains (MDRTB). Levels are much higher in those previously treated about $20 \%$. The frequency of MDR-TB varies substantially between countries. About $9 \%$ of MDR-TB cases also have resistance to two other classes of drugs, or extensively drug-resistant TB (XDRTB). By March 2013, 84 countries had reported at least one XDR-TB case [6]. MDRTB patient who fail treatment have a higher risk of death. Laboratory-confirmed RR-/MDR-TB cases in India are 25748 in 2014 with Patients started on MDR-TB treatment is 24073 [7]. MDR-TB cases among notified pulmonary TB cases 24000 among new cases i.e. $2.2 \%$ MDR-TB cases among notified pulmonary TB cases retreatment cases is $4700015 \%$ [8].

Very few studies have focused on the predicting factors for outcome of MDRTB treatment. In this study, we reviewed our experience with DRTB registered for dots-plus over a 48-month period at our hospital, a tertiary referred medical center, with special focus on the factors influencing the outcome of DRTB.

DRTB treatment programs have incorporated community participation in the DRTB treatment. Community-based directly observed therapy (cb-DOTS) programs are low-health care workers (HCWs) or former patients to directly observe treatment rather than requiring hospitalizations or frequent visits to a health care facility [911]. WHO recommended drugs and duration were for all regimens.

\section{Materials and Methods}

\section{Patient characteristics}

We retrospectively analyzed the medical records of 63 Patients with MDRTB from January 2011 to 2015 at Trichy General Hospital, a tertiary referred medical center in southern India. All the registered patients were followed by DOTS PLUS site with regular follow up investigation and vigil on adverse drug reactions. It is a Retrospective study and ethical clearance from institutional review committee obtained. MDRTB and XDRTB were included as DRTB Patients.

MDRTB: Defined as resistance to Isoniazid and Rifampicin.

*Corresponding author: Arivudainambi Periasamy, K.A.P.Viswanatham Government Medical College, Tiruchirappalli, India, Tel: +919443248165; E-mail: arivudain@yahoo.com

Received November 05, 2016; Accepted January 27, 2017; Published January 30, 2017

Citation: Periasamy A (2017) Predictors of Outcome in Drug Resistant Tuberculosis Patients. J Pulm Respir Med 7: 391. doi: 10.4172/2161-105X.1000391

Copyright: (๑ 2017 Periasamy A. This is an open-access article distributed under the terms of the Creative Commons Attribution License, which permits unrestricted use, distribution, and reproduction in any medium, provided the original author and source are credited. 
XDRTB: Defined as resistance to Isoniazid and Rifampicin and to $2^{\text {nd }}$ line drugs, Fluoroquinolones and Injectable.

PreXDRTB: Defined as resistance to Isoniazid and Rifampicin and either to any one of the $2^{\text {nd }}$ line drugs and Fluoroquinolones and Injectable.

\section{Data collection}

The following data were collected for each patient: age, gender, clinical symptoms, important underlying diseases, diabetes mellitus, Smoking history, Drug abuse, Alcoholism, CXR lesion characteristics, HIV coinfection, Drug culture and sensitivity of each patients (IRL). Outcome reported as success and failure. Success includes cured and treatment completed patients. Failure includes treatment failure, death, and defaulted, untraceable cases.

Cure: A patient who has completed treatment and has been consistently culture negative ( 5 consecutive negative results in the last 12 to 15 months) [12].

Treatment completed: A patient who has completed treatment but does not meet the criteria for cure or treatment failure due to lack of bacteriological results [13].

Treatment failure: Treatment will be considered to have failed if two or more of the five cultures recorded in the final five 12-15 months are positive, or if any of the final three culture are positive.

Death: A patient who dies for any reason during MDRTB/XDRTB treatment.

Treatment default: A patient whose treatment was interrupted for two or more consecutive months for any reasons.

Transfer out: A patient who has been transferred to another reporting unit and for whom the treatment outcome is not known.

\section{Statistical analysis}

The primary end of the present study was the success or failure of DRTB treatment. Possible predicting factors for the success or failure of therapy were assessed against this end. Pearson chi square test, $\chi^{2}$ (Fisher's Exact Test when needed) test was used for discrete data. One way ANOVA used for assessment of each variable effect on the outcome. Logistic regression analysis was applied to adjust for confounding variables to assess the possible predicting factors. All reported $\mathrm{p}$ values are two tailed, and a $\mathrm{p}$ value $<0.05$ was statistically significant. SPSS 21 was used for analysis.

\section{Results}

\section{Patients characteristics}

Sixty-three patients were included in the present study. The mean \pm SD age of the study population was 40.77 . Male gender was more frequent $46(73 \%)$ than female $17(27 \%)$. Out of total 63 cases, 33 cases had failed outcome (52.4\%) and 30 (47.6\%) cases had successful outcome. There were 7 patients (7.9\%) with HIV co-infection. There were $11(17.5 \%)$ diabetes patients in the cohort of MDRTB patients. There were 7 patients with Pre XDRTB among the 63 MDRTB cases. There were 37 patients who smoked either cigarettes or abused drugs. There were 32 patients who were alcoholic in cohort of 63 MDRTB patients.

Logistic regression analysis showed variables Diabetes, XDRTB suspects, drug abuse and smoking were associated with failed outcome. Sex, age, chest $\mathrm{x}$-ray lesion, pre-treatment resistance profile, did not affect the outcome.

\section{Binominal logistic regression}

By logistic regression (enter) method the odds of failure were 11.737 for smoking, the odds of failure were 12.43 for XDRTB suspects, the odds of failure with diabetes were 12.61. The P-value of smoking, XDRTB suspect, diabetes obtained were $0.019,0.049,0.036$ respectively (Table 1).

\section{Cox \& Snell R Square and Nagelkerke R Square}

These are pseudo R-squares their values were 0.314 and 0.419 . These values shows good fitness of the model. Hosmer and Lemeshow test had value of $\mathrm{P}=0.953$ establishing a good model (Table 2). Overall percentage of prediction of the model is 73 (Table 3 ).

\section{Chi-square test and univariate analysis}

$\chi^{2}(1)=7.931 \mathrm{P}=0.005$ for Diabetes mellitus.

$\chi^{2}(1)=11.503 \mathrm{P}=0.001$ for Smoking and drug abuse.

$\chi^{2}(1)=4.925 \mathrm{P}=0.026$ for Sex (gender) (Table 4).

Among 63MDRTB Patients 37 were smokers with 25 had failed outcome and 12 successful outcomes. Among 26 non-smoker MDRTB Patients only 8 had failed outcome.

Fisher exact test had significant P values for Diabetes mellitus,

\begin{tabular}{|c|c|c|c|c|c|c|c|c|}
\hline \multicolumn{9}{|l|}{ Binary logistic regression } \\
\hline \multirow[t]{2}{*}{ Variables } & \multicolumn{4}{|c|}{ Enter method } & \multicolumn{4}{|c|}{ Backward conditional method } \\
\hline & B & EXP B/ODDS & $\begin{array}{c}\text { (SIG) } \\
\text { P-Value }\end{array}$ & $\begin{array}{c}\text { Confidence } \\
\text { Interval }\end{array}$ & B & EXP B/ODDS & $\begin{array}{c}\text { (SIG) } \\
\text { P-Value }\end{array}$ & $\begin{array}{c}\text { Confidence } \\
\text { Interval }\end{array}$ \\
\hline Sex (Gender) & 0.666 & 1.946 & 0.553 & $0.216-17.504$ & 0.666 & 1.946 & 0.553 & $0.216-17.504$ \\
\hline XDRTB & 2.521 & 12.43 & 0.049 & $1.012-152.16$ & 2.487 & 12.024 & 0.049 & $1.012-142418$ \\
\hline Diabetes mmellitus & 2.535 & 12.619 & 0.036 & $1.185-134.89$ & 2.451 & 11.599 & 0.037 & $1.180-116.89$ \\
\hline Smoking and drugs abuse & 2.463 & 11.737 & 0.019 & $1.494-92.225$ & 2.003 & 7.415 & 0.020 & $2.024-27.158$ \\
\hline
\end{tabular}

Table 1: Model summary.

\begin{tabular}{|c|c|c|c|}
\hline Step & $\mathbf{- 2}$ Log likelihood & $\begin{array}{c}\text { Cox and Snell R } \\
\text { square }\end{array}$ & Nagelkerke R square \\
\hline 1 & $63.620^{\text {a }}$ & 0.312 & 0.417 \\
\hline \multicolumn{3}{|l}{ Hosmer and Lemeshow test } \\
\hline Step & Chi-square & df & Sig. \\
\hline 1 & 2.131 & 4 & 0.712 \\
\hline
\end{tabular}

$\mathrm{R}$ Square and Hosmer and Lemeshow test. Table 2: Cox and Snell R Square and Nagelkerke

\begin{tabular}{|l|c|c|c|}
\hline \multirow{2}{*}{ Observed } & \multicolumn{3}{|c|}{ Predicted } \\
\cline { 2 - 4 } & \multicolumn{2}{|c|}{ Outcome } & Percentage correct \\
\cline { 2 - 4 } & Failure & Success \\
\hline Outcome failure & 29 & 4 & 87.9 \\
\hline Success & 13 & 17 & 56.7 \\
\hline Overall percentage & \multicolumn{3}{|c|}{} \\
\hline
\end{tabular}

Table 3: Overall percentage of prediction. 
Smoking, Sex (gender). The P values (2 sided) for Diabetes mellitus, Smoking, Sex were 0.007, 0.001, 0.045 respectively (Table 4 ).

One way ANOVA showed Sex (gender), XDRTB, Diabetes, Smoking having significant $\mathrm{P}$ values rejecting the null hypothesis. Sex (gender), XDRTB, Diabetes, Smoking had P-values of 0.026, 0.05, 0.004, 0.000 respectively (Table 5). Variables such as HIV, Comorbidity, alcoholism, Chest $\mathrm{x}$-ray lesions like cavitary, caseous lesion did not have significant effect on the outcome (Table 6).

\section{Discussion and Conclusion}

Several hypotheses have been postulated to explain mechanisms that could lead to increased risk of TB in patients with DM, and increased risk of mortality in TB-DM patients. Both mouse and human models have demonstrated that DM alters adaptive and cell-mediated immune responses [14-16]. Impaired alveolar macrophage activation due to glycation of binding sites may inhibit subsequent granuloma formation in TB-DM patients [17]. In addition, altered T-helper (Th) 1 , Th 2, and Th 17 cytokine responses have been demonstrated among patients with TB-DM $[18,19]$. Chronic hyperglycemia may disrupt the regulation of key cytokines, such as interferon-gamma [16,20], which in turn may increase the $M$. tuberculosis bacterial burden and subsequent risk of death in TB-DM patients

Diabetes mellitus (DM) is an increasingly recognized comorbidity that can both accelerate TB disease and complicate TB treatment. The prevalence of DM among TB patients around the world varies according to different regions that range from 12 to $44 \%$ and tended to increase in the past decade [21]. It increases the risk of TB disease, complicates $\mathrm{TB}$ treatment, and increases the risk of a poor TB outcome [22,23]. Among MDR-TB patients, DM is a relatively common comorbidity [24]. In addition to the well-established contribution of DM to enhanced TB risk, there is growing evidence from observational studies that this comorbidity is associated with delays in mycobacterium TB clearance during treatment, treatment failures, death, relapse and reinfection [25]. However, whether DM presents any additional risk for the development or acquisition of MDR-TB remains controversial [26].
Three case-control studies comparing DM/TB and non-diabetic TB patients from Iran, Saudi Arabia, and Turkey showed no significant association between DM and the risk of MDR-TB [27-29]. Similarly, cross-sectional studies in Iran, Turkey, and Taiwan have reported no association between DM and MDR-TB [29-31]. On the other hand, many studies have found 2.1 to 8.8 times increased the risk of MDRTB among diabetic TB patients [19,32-35]. In addition, observational studies from Israel, Georgia, and Mexico have also shown patients with DM had a higher risk of developing MDR-TB $[14,15,36]$.

The 2014 U.S. Surgeon General's Report implicates smoking as a cause of TB disease among those latently infected with Mycobacterium tuberculosis (M. tb) [37]. While there is ample evidence that smoking increases the risk of TB and risk of death from TB [38], cell mediated immunity and macrophage function, essential to the host defense against $M$. tuberculosis infection, are directly impaired by exposure to tobacco smoking [12].

No other study had diabetes, PreXDRTB, smoking, as independent predicting factors of outcome of DRTB patients. Failure rate in resource limited country is not comparable to low burden country such as UK, U.S [39].

The results are like another study from high burden countries (South Africa) which had failure rate close to 60\% [40]. Similar study in US showed $78 \%$ success rate and death was limited to only $11 \%$. Even such study did not recommend pre XDRTB as outcome predictor, this study recommends preXDRTB to be treated as XDRTB [41]. The success rate in the series is $47.62 \%$ while the failure rate is $52.38 \%$ which is like the WHO global estimate which is $48 \%$ [42]. This is the only study on outpatient MDRTB treatment, other studies were all based on inpatient management of MDR patients [39,42].

In conclusion, our results showed PreXDRTB, Diabetes Mellitus Smoking and Drug abuse as independent predictors of failure. These results suggest that strict control of DM, switch over to XDRTB regime from MDRTB regimen in PreXDRTB is warranted, Smoking and drug

\begin{tabular}{|c|c|c|c|c|}
\hline \multirow[t]{2}{*}{ Variables } & Pearson chi square value and $P$-value & Fisher exact test (exact sig) & Likelihood ratio & Linear by linear \\
\hline & & & Value and $P$-value & association value and $P$-value \\
\hline \multirow[t]{2}{*}{ Diabetes mellitus } & 7.931 & 0.007 & 9.098 & 7.865 \\
\hline & $P=0.005$ & & $P=0.003$ & $P=0.005$ \\
\hline \multirow[t]{2}{*}{ Smoking and drug abuse } & 11.503 & 0.001 & 11.871 & 11.32 \\
\hline & $P=0.001$ & & $P=0.001$ & $P=0.001$ \\
\hline \multirow[t]{2}{*}{ Sex (Gender) } & 4.925 & 0.045 & 5.018 & 4.846 \\
\hline & $P=0.026$ & & $P=0.025$ & $P=0.028$ \\
\hline
\end{tabular}

Table 4: Chisquare test.

\begin{tabular}{|l|c|c|c|}
\hline \multicolumn{1}{|c|}{ Variables } & \multicolumn{2}{c|}{ ANOVA } & \multirow{2}{*}{ Robust tests of equality of means. Welch P value } \\
\cline { 2 - 3 } & F value & 0.026 \\
\hline Sex (Gender) & 5.173 & 0.053 & \\
\hline XDRTB & 3.597 & 0.027 \\
\hline Diabetes mellitus & 8.785 & 0.004 & 0.001 \\
\hline Smoking and drug abuse & 13.625 & 0.000 & 0.001 \\
\hline
\end{tabular}

Table 5: One way ANOVA.

\begin{tabular}{|l|c|c|c|}
\hline \multirow{2}{*}{ Variables } & \multicolumn{2}{|c|}{ ANOVA } & \multirow{2}{*}{ Robust tests of equality of means. Welch P value } \\
\cline { 2 - 3 } & F value & 0.727 & \\
\hline HIV & 0.123 & 0.09 & 0.759 \\
\hline Comorbidity & 2.95 & 0.09 & 0.106 \\
\hline Alcoholism & 2.699 & 0.160 & 0.190 \\
\hline Radiological lesion (cavitary, caseous, nodular) & 1.897 & & \\
\hline
\end{tabular}

Table 6: One way ANOVA. 
rehabilitation to be carried out in DRTB patients. Non-communicable disease effect must be studied extensively.

\section{Limitation}

It is a retrospective study. Data such as glycemic control prior to diagnosis of MDRTB could not be accurately known. There are important limitations to note in our study. First, we relied on selfreport and medical chart abstraction to determine whether TB patients had DM, and therefore the primary exposure of interest was subject to misclassification due to TB patients who did not know they had DM or who had never been screened.

\section{References}

1. Gandhi NR, Nunn P, Dheda K, Schaaf HS, Zignol M, et al. (2010) Multidrugresistant and extensively drug-resistant tuberculosis: a threat to global control of tuberculosis. Lancet 375: 1830-1843.

2. WHO (2011) World Health Organization. Global Plan to Stop TB 2011-2015 Geneva.

3. Laserson KF, Thorpe LE, Leimane V, Weyer K, Mitnick CD, et al. (2005) Speaking the same language: treatment outcome definitions for multidrugresistant tuberculosis. Int J Tuberc Lung Dis 14: 640-645.

4. WHO (2011) Guidelines for Programmatic Management of Drug-Resistan Tuberculosis (2011 Update) Geneva.

5. http://www.who.int/tb/challenges/mdr/MDR_TB_FactSheet.pdf

6. World Health Organization, India /prolife 2013.(who.int/tb/country/data/profiles/in)

7. World Health Organization Estimates of TB and MDR-TB burden are produced by WHO in consultation with countries.(who.int/tb/country/data/profiles/in)

8. Okello D, Floyd K, Adatu F, Odeke R, Gargioni G (2003) Cost and costeffectiveness of community-based care for tuberculosis patients in rural Uganda. Int J Tuberc Lung Dis 7: S72-S79.

9. Wandwalo E, Kapalata N, Egwaga S, Morkve O (2004) Effectiveness of community-based directly observed treatment for tuberculosis in an urban setting in Tanzania: a randomised controlled trial. Int J Tuberc Lung Dis 14 1248-1254.

10. Falzon D, Jaramillo E, Schünemann HJ, Arentz M, Bauer M, et al. (2011) WHO guidelines for the programmatic management of drug-resistant tuberculosis: 2011 update. Eur Respir J 38: 516-528.

11. Adatu F, Odeke R, Mugenyi M, Gargioni G, McCray E, et al. (2003) Implementation of the DOTS strategy for tuberculosis control in rural Kiboga District, Uganda, offering patients the option of treatment supervision in the community, 1998-1999. Int J Tuberc Lung Dis 14: S63-S71.

12. Marks SM, Flood J, Seaworth B, Hirsch-Moverman Y, Armstrong L, et al. (2014) Treatment practices, outcomes, and costs of multidrug-resistant and extensively drug-resistant tuberculosis, United States, 2005-2007. Emerg Infect Dis 20: 812-821.

13. Pieters $J$ (2008) Mycobacterium tuberculosis and the macrophage: maintaining a balance. Cell Host Microbe 3: 399-407.

14. Restrepo BI, Fisher-Hoch SP, Pino PA, Salinas A, Rahbar MH, et al. (2008) Tuberculosis in poorly controlled type 2 diabetes: altered cytokine expression in peripheral white blood cells. Clin Infect Dis 47: 634-641.

15. Stalenhoef JE, Alisjahbana B, Nelwan EJ, van der Ven-Jongekrijg J, Ottenhoff $\mathrm{TH}$, et al. (2008) The role of interferon-gamma in the increased tuberculosis risk in type 2 diabetes mellitus. Eur J Clin Microbiol Infect Dis 27: 97-103.

16. Banerjee D, Bhattacharyya R, Kaul D, Sharma P (2011) Diabetes and tuberculosis: analysis of a paradox. Adv Clin Chem 53: 139-153.

17. Dooley KE, Tang T, Golub JE, Dorman SE, Cronin W (2009) Impact of diabetes mellitus on treatment outcomes of patients with active tuberculosis. Am J Trop Med Hyg 80: 634-639.

18. Kleinbaum D, Klein M (2012) Survival Analysis: A Self-Learning Text. (3rd edn) New York: Springer.

19. Moutschen MP, Scheen AJ, Lefebvre PJ (1992) Impaired immune responses in diabetes mellitus: analysis of the factors and mechanisms involved. Relevance to the increased susceptibility of diabetic patients to specific infections. Diabete Metab 18: 187-201.

20. Porkert MT, Sotir M, Parrott-Moore P, Blumberg HM (1997) Tuberculous meningitis at a large inner-city medical center. Am J Med Sci 313: 325-331.

21. Kourbatova EV, Leonard MK Jr, Romero J, Kraft C, del Rio C, et al. (2006) Risk factors for mortality among patients with extrapulmonary tuberculosis at an academic inner-city hospital in the U.S. Eur J Epidemiol 21: 715-721.

22. Sterling TR, Zhao Z, Khan A, Chaisson RE, Schluger N, et al. (2006) Mortality in a large tuberculosis treatment trial: modifiable and non-modifiable risk factors. Int J Tuberc Lung Dis 10: 542-549.

23. Kourbatova EV, Borodulin BE, Borodulina EA, del Rio C, Blumberg HM, et al. (2006) Risk factors for mortality among adult patients with newly diagnosed tuberculosis in Samara, Russia. Int J Tuberc Lung Dis 10: 1224-1230.

24. Walpola HC, Siskind V, Patel AM, Konstantinos A, Derhy P (2003) Tuberculosisrelated deaths in Queensland, Australia, 1989-1998: characteristics and risk factors. Int J Tuberc Lung Dis 7: 742-750.

25. Raviglione M, Marais B, Floyd K, Lonnroth K, Getahun H, et al. (2012) Scaling up interventions to achieve global tuberculosis control: progress and new developments. Lancet 379: 1902-1913.

26. Dooley KE, Chaisson RE (2009) Tuberculosis and diabetes mellitus: convergence of two epidemics. Lancet Infect Dis 9: 737-746.

27. Baker MA, Harries AD, Jeon CY, Hart JE, Kapur A, et al. (2011) The impact of diabetes on tuberculosis treatment outcomes: a systematic review. BMC Med 9: 81.

28. Oursler KK, Moore RD, Bishai WR, Harrington SM, Pope DS, et al. (2002 Survival of patients with pulmonary tuberculosis: clinical and molecular epidemiologic factors. Clin Infect Dis 34: 752-759.

29. Restrepo BI, Fisher-Hoch SP, Crespo JG, Whitney E, Perez A, et al. (2007) Type 2 diabetes and tuberculosis in a dynamic bi-national border population. Epidemiol Infect 135: 483-491.

30. GDPH (2011) Georgia Tuberculosis Report. Atlanta: Georgia Department of Public Health.

31. Greenland S, Pearl J, Robins JM (1999) Causal diagrams for epidemiologic research. Epidemiology 10: 37-48.

32. IDF (2012) Diabetes Atlas, Update 2012. Brussels: International Diabetes Federation.

33. Oluboyo PO, Erasmus RT (1990) The significance of glucose intolerance in pulmonary tuberculosis. Tubercle 71: 135-138.

34. Niemi M, Backman JT, Neuvonen M, Neuvonen PJ, Kivistö KT (2001) Effects of rifampin on the pharmacokinetics and pharmacodynamics of glyburide and glipizide. Clin Pharmacol Ther 69: 400-406.

35. Kapur A, Harries AD (2013) The double burden of diabetes and tuberculosis public health implications. Diabetes Res Clin Pract 101: 10-19.

36. ASPA (2014) The Health Consequences of Smoking-50 Years of Progress: A Report of the Surgeon General

37. Sitas F, Urban M, Bradshaw D, Kielkowski D, Bah S, et al. (2004) Tobacco attributable deaths in South Africa. Tob Control 13: 396-399.

38. Lin H-H, Ezzati M, Murray M (2007) Tobacco smoke, indoor air pollution and tuberculosis: A systematic review and meta-analysis. PLoS Med 4: e20.

39. WHO (2008) Guidelines for the programmatic management of drug-resistant tuberculosis. Emergency update 2008. Geneva: World Health Organization.

40. (2003) The PIH guide to the medical management of multidrug-resistant tuberculosis. Boston, MA: PIH.

41. Marais E, Mlambo CK, Lewis JJ, Rastogi N, Zozio T, et al. (2014) Treatment outcomes of multidrug-resistant tuberculosis patients in Gauteng, south Africa. Infection 42: 405-413.

42. Anderson LF, Tamne S, Watson JP, Cohen T, Mitnick C, et al. (2013) Treatment outcome of multi-drug resistant tuberculosis in the United Kingdom: retrospective-prospective cohort study from 2004 to 2007. Euro Surveill 18: 20601. 